\title{
Suboptimal Event-based Control of Linear Systems over Lossy Channels *
}

\author{
Adam Molin* Sandra Hirche* \\ * Institute of Automatic Control Engineering, Technische Universität \\ München, D-80290 München, Germany (e-mail: adam.molin@tum.de, \\ hirche@tum.de).
}

\begin{abstract}
The emergence of networked control systems urges the joint consideration of control and communications. It is well known that event-based controllers outperform standard timetriggered designs, when the transmission rate is constrained. This paper considers the design of suboptimal event-based controllers in the presence of packet loss and delayed acknowledgement for linear stochastic systems. The design objective consists of a quadratic cost reflecting the control performance and a communication penalty term. Based on various restrictions on the control design, optimal solutions within the corresponding class of event-based controller are derived. A numerical validation illustrates the effectiveness of the proposed event-triggers and gives a comparison to the optimal time-triggered controller.
\end{abstract}

Keywords: Networked control systems, control over communications, event-based control, stochastic optimal control

\section{INTRODUCTION}

Digital control systems are commonly designed so that measurements are acquired periodically to update actuator inputs. With the advent of networked control systems various benefits emerged by separating sensors, actuators and controllers into self-contained digital devices with communicating capabilities. Those benefits including flexibility, robustness and reconfigurability, come at the price of new design challenges due to constraints imposed by the digital communication system. It has been already indicated in the literature comprising Åström and Bernhardsson (2002); Cervin and Henningsson (2008), that event-triggered control systems outperform standard timetriggered sampled systems, when transmission rate constraints are incorporated. The work in Åström and Bernhardsson (2002) shows that an event-triggered impulsive controller is capable of reducing the state variance significantly compared to a time-triggered minimum variance controller, while both having the same average transmission rate. In Cervin and Henningsson (2008), multiple independent control loops share a common digital network. There, it turns out that an event-triggered scheduling scheme outperforms a time-triggered scheduling scheme in terms of aggregate state variance.

The occurrence of time-delay and packet dropouts in digital communication networks is unavoidable and needs to be considered by the designer of the networked control system. Most results on the design of optimal eventbased controllers neglect these shortcomings and only few works incorporate time-delay and packet dropouts within their design. In Rabi and Johansson (2009) the design

\footnotetext{
^ This work was supported in part by the German Research Foundation (DFG) within the Priority Program SPP 1305 "Control Theory of Digitally Networked Dynamical Systems".
}

of optimal level-triggered impulse control under packet dropouts with multiple loops sharing a common network is considered. The work in Bommannavar and Başar (2008) derives the optimal event-based controller in the presence of packet dropouts and under a constraint on the number of transmissions. In Xu and Hespanha (2004), optimal event-based estimators are designed by imposing a penalty on sending updates over a communication network with fixed delay.

The main contribution of this paper is to develop optimal event-based controllers under time-delay and packet dropouts in the feedback link. The presence of both of these degrading sources poses the need for an innovative event-based control design. By assuming that the digital communication system provides an acknowledgement mechanism, this paper gives two different design guidelines for event-based controllers within a stochastic discretetime linear framework. The objective function is inspired by work in $\mathrm{Xu}$ and Hespanha (2004). It consists of a quadratic cost representing the control performance and a communication cost penalizing update transmissions over the channel. Except for the special case of undelayed acknowledgements, it is very hard to find the optimal eventbased controller minimizing the objective function, as indicated in Molin and Hirche (2009). This fact motivates us to propose optimal event-based controllers within a certain class of controllers. The first class prohibits the eventtrigger to transmit an update, if there is an outstanding acknowledgement, whereas the second class finds the optimal event-trigger for a fixed controller at the actuator. The results are based on novel reformulation techniques developed in Molin and Hirche (2009) and recent generalizations of state estimation for Markovian jump linear systems with delayed observation derived in Matei et al. (2008). A numerical validation shows that the proposed 
suboptimal algorithms approach a lower bound of the cost function very closely and outperform the optimal timetriggered event-based controller.

This paper is organized into four sections. Section 2 gives the problem statement. In section 3, two suboptimal approaches are proposed for the underlying problem and their solutions are derived. The efficacy of the suboptimal algorithms is validated in section 4 .

Notation. In this paper, the operators $\operatorname{tr}[\cdot]$ and $(\cdot)^{\mathrm{T}}$ denote the trace and the transpose operator of a square matrix, respectively. The symbol ' $\wedge$ ' denotes the logical AND-operation. The operator $\mathbb{1}_{(\cdot)}$ denotes the indicator function. The expectation operator is denoted by $\mathrm{E}[\cdot]$ and the conditional expectation is denoted by $\mathrm{E}[\cdot \mid \cdot]$. Sans-serif variables, e.g. $x_{k}$, indicate realizations of random variables. A sequence of a random process $\left\{x_{k}\right\}_{k}$ is denoted by $\boldsymbol{X}^{k}=\left[x_{0}, \ldots, x_{k}\right]$ for its complete history and $\boldsymbol{X}_{l}^{k}=\left[x_{l}, \ldots, x_{k}\right]$ for a specific time interval $\{l, \ldots, k\}$. If $l>k$, then $\boldsymbol{X}_{l}^{k}$ is the empty set.

\section{PROBLEM FORMULATION}

We consider the following stochastic time-invariant discretetime system $\mathcal{P}$

$$
x_{k+1}=A x_{k}+B u_{k}+w_{k},
$$

where $A \in \mathbb{R}^{n \times n}, B \in \mathbb{R}^{n \times d}$. The variables, $x_{k}$ and $u_{k}$ denote the state and the control input. They are taking values in $\mathbb{R}^{n}$ and $\mathbb{R}^{d}$, respectively. The system noise $w_{k}$ takes values in $\mathbb{R}^{n}$ and is an i.i.d. (independent identically distributed) zero-mean Gaussian distributed sequence with covariance matrix $\Sigma_{w}$. The initial state, $x_{0}$ is Gaussian with mean $m_{0}$ and covariance $\Sigma_{x_{0}}$.

The system model for the communication system is given by an erasure channel in the forward link. Packet dropouts are modeled as a Bernoulli process $\left\{q_{k}\right\}_{k}$ defined as

$$
q_{k}= \begin{cases}1 & \text { update successfully transmitted } \\ 0 & \text { packet dropout occurred }\end{cases}
$$

with packet dropout probability $\beta=\mathrm{P}\left[q_{k}=0\right]$. We assume a TCP-like communication protocol as introduced in Schenato et al. (2007) for networked control systems. The main feature of TCP-like communication protocols is that a binary acknowledgement is sent over the reverse link to the event-trigger, whenever a packet has been transmitted successfully. It is assumed that the reverse link is error-free. Forward and reverse link delay packets by the duration of $T_{1}$ and $T_{2}$, respectively. Both, $T_{1}$ and $T_{2}$, are positive and non-negative integer values. Let $(\Omega, \mathcal{A}, \mathrm{P})$ denote the probability space generated by the random variables $x_{0},\left\{w_{k}\right\}_{k}$ and $\left\{q_{k}\right\}_{k}$. System parameters and statistics are known to the event-trigger and controller. The event-trigger $\mathcal{E}$ situated at the sensor side has access to the complete observation history and decides, whether the controller $\mathcal{C}$ should receive an update. The system model is illustrated in Fig. 1.

The event-trigger output $\delta_{k} \in\{0,1\}$ is defined as follows:

$$
\delta_{k}= \begin{cases}1 & \text { update is sent } \\ 0 & \text { nothing transmitted }\end{cases}
$$

If the event-trigger decides to update the controller, it transmits the current state over the erasure channel to the controller. The input signal at the controller is written as:

$$
y_{k+T_{1}}= \begin{cases}x_{k}, & \delta_{k}=1 \wedge q_{k}=1 \\ \emptyset, & \text { otherwise }\end{cases}
$$

with $y_{0}=\cdots=y_{T_{1}-1}=\emptyset$.

The design objective is to find admissible event-triggering policies $\mathrm{f}$ and control policies $\gamma$ that minimize the finitehorizon criterion

$$
J(\mathrm{f}, \gamma)=\mathrm{E}\left[x_{N}^{\mathrm{T}} Q_{N} x_{N}+\sum_{k=0}^{N-1} x_{k}^{\mathrm{T}} Q x_{k}+u_{k}^{\mathrm{T}} R u_{k}+\lambda \delta_{k}\right] .
$$

The weighting matrices $Q, Q_{N}$ are positive definite and $R$ is positive semi-definite. The positive factor $\lambda$ can be regarded as the weight of penalizing information exchange between sensor and controller. Admissible policies for the event-trigger and the controller at time $k$ are defined as Borel-measurable functions of their past available data

$$
\delta_{k}=\mathrm{f}_{k}\left(\mathcal{I}_{k}^{\mathcal{E}}\right), \quad u_{k}=\gamma_{k}\left(\mathcal{I}_{k}^{\mathcal{C}}\right)
$$

The information patterns $\mathcal{I}_{k}^{\mathcal{E}}$ and $\mathcal{I}_{k}^{\mathcal{C}}$ of the event-trigger and controller are defined as

$$
\mathcal{I}_{k}^{\mathcal{E}}=\left\{\boldsymbol{X}^{k}, \boldsymbol{\delta}^{k-1}, \boldsymbol{Q}^{k-T_{1}-T_{2}}\right\}, \quad \mathcal{I}_{k}^{\mathcal{C}}=\left\{\boldsymbol{Y}^{k}, \boldsymbol{U}^{k-1}\right\} .
$$

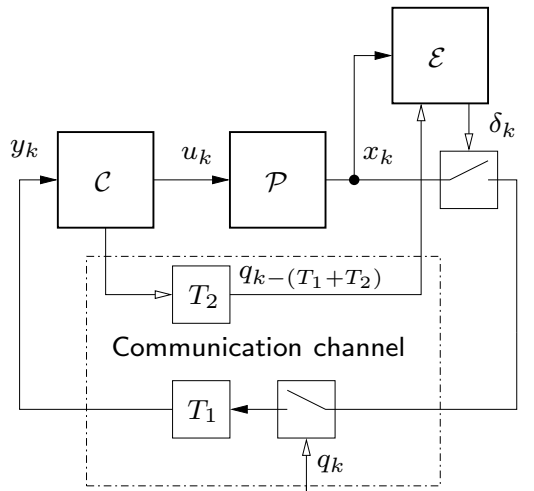

Fig. 1. System model of the networked control system with plant $\mathcal{P}$, event-trigger $\mathcal{E}$, controller $\mathcal{C}$ and a communication system. The forward link is an erasure channel and the reverse link carries the acknowledgement. Both links contain transmission delays $T_{1}, T_{2}$.

\section{SUBOPTIMAL EVENT-BASED CONTROLLERS}

Finding the optimal event-based controller is a hard problem for the underlying problem setting given by (3). The controller and event-trigger have different information patterns and it is well known by Witsenhausen (1968) that such problems are generally very difficult to solve. The situation is even worsened, as reformulation techniques developed in Molin and Hirche (2009) are not applicable due to the delay in the acknowledgement channel.

Due to these facts, we propose two types of suboptimal event-based controllers in the following. The term suboptimal refers to the optimal event-based controller under certain restrictions within the design. First, we propose a waiting strategy, where the event-trigger is prohibited to transmit updates, until the acknowledgement of the last transmission has been received. In the second approach, we prespecify the control law $\gamma$ and derive the optimal event-trigger. The prespecified controller is chosen to be a certainty equivalence controller. The approach is called 
dropout estimation strategy, as it involves the estimation, whether a packet dropout occurred before receiving the acknowledgement.

\subsection{Waiting Strategy}

The motivation for the waiting strategy is two-fold. On the one hand, it is reasonable to wait for the acknowledgement as it enhances predictability of the event-trigger for the communication system. On the other hand, the calculation of the optimal policy within this class turns out to be a tractable problem. This is enabled by separating the problem into numerically feasible subproblems with similar arguments as in Molin and Hirche (2009). In order to show such a property, we first reformulate the problem, so that optimal policies implicitly use the waiting strategy. We define an additional state variable $s_{k}$ given by

$$
s_{k+1}= \begin{cases}T_{1}+T_{2}-1 & \delta_{k}=1 \wedge s_{k}=0 \\ s_{k}-1 & \delta_{k}=0 \wedge s_{k}>0 \\ 0 & \delta_{k}=0 \wedge s_{k}=0\end{cases}
$$

with $s_{0}=0$ and the following interconnection relation which differs from Eq. (2)

$$
y_{k+T_{1}}= \begin{cases}x_{k}, & \delta_{k}=1 \wedge q_{k}=1 \wedge s_{k}=0 \\ \emptyset, & \text { otherwise. }\end{cases}
$$

Eq. (5) implies that even if $\delta_{k}=1$, the update will be blocked, when $s_{k}>1$. This reflects exactly the behavior of the waiting strategy, as choosing $\delta_{k}=1$ when $s_{k}>0$, will have no effect on the system evolution, although the communication penalty $\lambda$ is paid. Therefore, the optimal event-triggering law always selects $\delta_{k}=0$ for $s_{k}>0$.

In order to derive the optimal policies, we first consider the following related problem: First, assume that there is an additional side information channel that transmits the control input $u_{k-1}$ and the dropout variable $q_{k-T_{1}}$ instantaneously to the event trigger $\mathcal{E}$. Hence, the sequences $\boldsymbol{U}^{k-1}$ and $\boldsymbol{Q}_{k+1-\left(T_{1}+T_{2}\right)}^{k-T_{1}}$ are part of the information pattern at the event-trigger at time $k$ in the following. We will observe later that this additional side information is redundant for the optimal waiting strategy. Second, consider the following prespecified event-trigger that is constructed by an admissible event-trigger law $\overline{\mathrm{f}}$ and transformation $\mathcal{T}_{\bar{\gamma}}$ parameterized by an admissible control law $\bar{\gamma}$. The prespecified event-trigger is denoted by $\bar{f} \circ \mathcal{T}_{\bar{\gamma}}$. We seek for the optimal control law minimizing the cost given by Eq. (3) for given event-trigger $\bar{f} \circ \mathcal{T}_{\bar{\gamma}}$. Transformation $\mathcal{T}_{\bar{\gamma}}$ recalculates the state, received update information and control input that would result, if control policy $\bar{\gamma}$ would have been used. These variables are denoted by $\bar{x}_{k}, \bar{y}_{k}$ and $\bar{u}_{k}$, respectively. It can be shown that such transformation is given by

$$
\begin{aligned}
& \bar{x}_{k}=x_{k}+\sum_{m=0}^{k-1} A^{k-m-1} B\left(\gamma_{m}\left(\bar{y}_{0}, \ldots, \bar{y}_{m}\right)-u_{m}\right), \\
& \bar{u}_{k}=\bar{\gamma}_{k}\left(\bar{u}_{0}, \bar{y}_{0}, \ldots, \bar{u}_{k-1}, \bar{y}_{k}\right)
\end{aligned}
$$

The resulting variables are then used by the event-trigger law $\overline{\mathrm{f}}_{k}$ to calculate $\delta_{k}$.

The key feature of this related problem is that the sequence $\left\{\delta_{k}\right\}_{k}$ is independent of the control inputs chosen for each sample path $\omega \in \Omega$. With this fact, we can give the following lemma.
Lemma 1. Let the event-trigger $\bar{f} \circ \mathcal{T}_{\bar{\gamma}}$ be fixed with augmented information pattern $\mathcal{I}_{k}^{\mathcal{E}} \cup \boldsymbol{U}^{k-1} \cup \boldsymbol{Q}_{k+1-(T)}^{k-T_{1}}$ Then, the optimal control policy $\gamma^{*}$ minimizing cost function $J$ from (3) is given by

with

$$
u_{k}=\gamma_{k}^{*}\left(\mathcal{I}_{k}^{\mathcal{C}}\right)=-L_{k} \mathrm{E}\left[x_{k} \mid \mathcal{I}_{k}^{\mathcal{C}}\right]
$$

$$
\begin{aligned}
L_{k}= & \left(R+B^{\mathrm{T}} P_{k+1} B\right)^{-1} B^{\mathrm{T}} P_{k+1} A, \\
P_{k}= & A^{\mathrm{T}} P_{k+1} A+Q-A^{\mathrm{T}} P_{k+1} B \\
& \times\left(R+B^{\mathrm{T}} P_{k+1} B\right)^{-1} B^{\mathrm{T}} P_{k+1} A, \\
P_{N}= & Q_{N},
\end{aligned}
$$

where $P_{k} \in \mathbb{R}^{n \times n}$ is non-negative definite for all $k$.

Proof. Due to the fact that the sequence $\left(\delta_{k}\right)_{k}$ is independent of the sequence of control inputs for each sample path $\omega \in \Omega$, the term $\mathrm{E}\left[\sum_{k=0}^{N-1} \lambda \delta_{k}\right]$ in Eq. (3) is constant and can be omitted from the optimization. Similarly to Molin and Hirche (2009), it can be shown that $e_{k}=x_{k}-\mathrm{E}\left[x_{k} \mid \mathcal{I}_{k}^{\mathcal{C}}\right]$ is independent of applied controls. Proceeding along the same lines as in Bertsekas (2005), the resulting optimal control law is given by the linear quadratic regulator and the least squares estimate of the state as stated in above lemma.

Lemma 1 holds for any prespecified event-trigger $\bar{f} \circ \mathcal{T}_{\bar{\gamma}}$ constructed by the tuple $(\bar{\gamma}, \bar{f})$. It is clear that the policy pair $\gamma^{*}$ and $\bar{f} \circ \mathcal{T}_{\bar{\gamma}}$ are only optimal within a subset of admissible policy pairs $(\gamma, f)$. In fact, this subset can be viewed as an equivalence class defined by the following equivalence relation. Two policies, $\left(\gamma^{1}, f^{1}\right)$ and $\left(\gamma^{2}, f^{2}\right)$, are equivalent, if and only if

$$
\left(\delta_{k}^{1}\right)_{k}=\left(\delta_{k}^{2}\right)_{k}, \quad \omega \in \Omega .
$$

A fundamental property of equivalence classes is that the complete solution space of admissible policies is partitioned by the equivalence relation. As the form of the optimal controller does not depend on the specific equivalence class, we conclude that $\gamma^{*}$ given by (7) is the optimal control law for our original problem under the assumption of additional information $\boldsymbol{U}^{k-1} \cup \boldsymbol{Q}_{k+1-\left(T_{1}+T_{2}\right)}^{k-T_{1}}$ at the eventtrigger. This result enables us to restate the optimization problem (3). By using an identity from Åström (2006) the cost function $J$ defined in $(3)$ can be written as

$$
\begin{aligned}
& J(\mathrm{f}, \gamma)=\mathrm{E}\left[x_{0}^{\mathrm{T}} P_{0} x_{0}+\sum_{k=0}^{N-1} w_{k}^{\mathrm{T}} P_{k+1} w_{k}\right. \\
& \left.+\sum_{k=0}^{N-1}\left(u_{k}+L_{k} x_{k}\right)^{\mathrm{T}}\left(R+B^{\mathrm{T}} P_{k+1} B\right)\left(u_{k}+L_{k} x_{k}\right)+\lambda \delta_{k}\right],
\end{aligned}
$$

where $L_{k}$ and $P_{k}$ are given by Lemma 1 . Taking into account the optimal control law $\gamma^{*}$ given by (7), we obtain the following optimization problem

$$
J^{\mathcal{E}}(f)=\min _{f} \mathrm{E}\left[\sum_{k=0}^{N-1}\left(x_{k}-\hat{x}_{k}^{\mathcal{C}}\right)^{\mathrm{T}} \Gamma_{k}\left(x_{k}-\hat{x}_{k}^{\mathcal{C}}\right)+\lambda \delta_{k}\right],
$$

where

$$
\begin{aligned}
\hat{x}_{k}^{\mathcal{C}} & =\mathrm{E}\left[x_{k} \mid \mathcal{I}_{k}^{\mathcal{C}}\right] \\
\Gamma_{k} & =L_{k}^{\mathrm{T}}\left(R+B^{\mathrm{T}} P_{k+1} B\right) L_{k}, \quad k=0, \ldots, N-1 .
\end{aligned}
$$

As $\mathrm{E}\left[x_{0}^{\mathrm{T}} P_{0} x_{0}+\sum_{k=0}^{N-1} w_{k}^{\mathrm{T}} P_{k+1} w_{k}\right]$ is constant, this term has been omitted from the optimization. 
It should be noted that the state estimate $\hat{x}_{k}^{\mathcal{C}}$ depends on the chosen scheduling policy $\mathrm{f}$. Therefore, the dynamic programming principle can not be applied to solve problem (8). Proceeding along the same lines as in Molin and Hirche (2009), it can be shown that the optimal scheduling law possesses certain symmetry properties. These imply that the least-squares estimate $\hat{x}_{k}^{\mathcal{C}}$ is given by the leastsquares estimate assuming a time-triggered transmission scheme, i.e.

$\hat{x}_{k+T_{1}}^{\mathcal{C}}=\left\{\begin{array}{l}A^{T_{1}} x_{k}-\sum_{m=0}^{T_{1}-1} A^{T_{1}-m-1} B L_{k+m} \hat{x}_{k+m}^{\mathcal{C}}, \text { for } \delta_{k}=1 \wedge q_{k}=1 \wedge s_{k}=0, \\ \left(A-B L_{k}\right) \hat{x}_{k+T_{1}-1}^{\mathcal{C}}, \quad \text { otherwise }\end{array}\right.$

with initial conditions

$$
\hat{x}_{k}^{\mathcal{C}}=\left(A-B L_{k}\right) \cdots\left(A-B L_{0}\right) m_{0}, k=0, \ldots T_{1}-1 .
$$

By now, we have assumed that the event-trigger has additional information $\boldsymbol{U}^{k-1}$ and $\boldsymbol{Q}^{k-T_{1}}$ at time $k$. In the following, we will argue that this information is not needed for the optimal event-triggering law. Without loss of generality, we consider time step $k_{1}$ and assume there were no transmissions beforehand, i.e. $\boldsymbol{\delta}^{k_{1}-1}=[0, \ldots, 0]^{\mathrm{T}}$. By Eq. (10), we observe that

$$
\hat{x}_{k}^{\mathcal{C}}=\left(A-B L_{k}\right) \cdots\left(A-B L_{0}\right) m_{0}, \quad k=0, \ldots, k_{1} .
$$

This implies that the dropout sequence $\boldsymbol{Q}^{k_{1}-1}$ has no impact on the state estimates $\hat{\boldsymbol{X}}^{\mathcal{C}, k_{1}}$ and the control inputs $\boldsymbol{U}^{k_{1}-1}$. Therefore, the event-trigger does not need to take into account the dropout sequence $\boldsymbol{Q}^{k_{1}-1}$ and control inputs $\boldsymbol{U}^{k_{1}-1}$ can be recalculated by the eventtrigger with information pattern $\mathcal{I}_{k_{1}}^{\mathcal{E}}$. If it has been decided that $\delta_{k_{1}}=1$, then the event-trigger is idle for the duration of $T_{1}+T_{2}-1$ and no information is needed during this phase. At time step $k_{1}+T_{1}+T_{2}$, the acknowledgement $q_{k_{1}}$ is received by the event-trigger. Hence, the state estimate $\hat{x}_{k_{1}+1}^{\mathcal{C}}$ and $u_{k_{1}+1}$ can be calculated with information pattern $\mathcal{I}_{k_{1}}^{\mathcal{E}}$. By induction, it follows that additional information $\boldsymbol{U}^{k-1}$ and $\boldsymbol{Q}^{k-T_{1}}$ is not needed for the optimal event-triggering policy at any time step $k$.

In order to facilitate further illustration of results, we consider $T_{1}$ to be 1 in the following. By considering Eqs. (1), (4), (7) and (10), we define the estimation error $e_{k}=x_{k}-\hat{x}_{k}^{\mathcal{C}}$, which transforms problem (8) into

$$
\begin{aligned}
& \min _{f} \mathrm{E}\left[\sum_{k=0}^{N-1} e_{k}^{\mathrm{T}} \Gamma_{k} e_{k}+\lambda \delta_{k}\right], \\
& e_{k+1}=\left(1-\mathbb{1}_{\left\{s_{k}=0\right\}} q_{k} \delta_{k}\right) A e_{k}+w_{k}
\end{aligned}
$$

with initial conditions $e_{0}=x_{0}-m_{0}$ and $s_{k}$ given by (4). The optimal event-triggering policy will be a function of the augmented state $\left[e_{k}, s_{k}\right]$ and the optimization problem (12) can be solved by the dynamic programming principle. Similar results also apply for arbitrary $T_{1}$.

Hence, the main result of this section is that the optimal event-based controller within the waiting strategy can be solved by standard methods. The controller is linear and the gains are obtained by the Riccati equation, while the state estimator is a linear predictor. The event-triggering policy can be obtained by dynamic programming.

\subsection{Dropout Estimation Strategy}

The proposed event-based controller in the last section is certainly suboptimal, as there are circumstances, where another update should be sent, although the outstanding acknowledgement has not been received yet. For example, this would be the case, if a significant state disturbance is observed, while the event-trigger has to wait. This fact motivates us to relax the waiting strategy and allow update transmissions before an outstanding acknowledgement has been received. In the following, we restrict the control law to be predetermined by the optimal control law of the waiting strategy. In other words, the control law is the certainty equivalence controller given by Lemma 1 . Under this assumption, our focus is to find the event-triggering policy that solves problem (8).

The control law is given by Eq. (7) and, similarly to Eq. (10), the state estimate is given by

$$
\hat{x}_{k+T_{1}}^{\mathcal{C}}=\left\{\begin{array}{l}
A^{T_{1}} x_{k}-\sum_{m=0}^{T_{1}-1} A^{T_{1}-m-1} B L_{k+m} \hat{x}_{k+m}^{\mathcal{C}}, \text { for } \delta_{k}=1 \wedge q_{k}=1, \\
\left(A-B L_{k}\right) \hat{x}_{k+T_{1}-1}^{\mathcal{C}}, \quad \text { otherwise }
\end{array}\right.
$$

with initial conditions given by Eq. (11). Above state estimator differs only by its independence of $s_{k}$. We define

$$
z_{k}=\left[x_{k}^{\mathrm{T}}, \hat{x}_{k}^{\mathcal{C}, \mathrm{T}}, \ldots, \hat{x}_{k+T_{1}-1}^{\mathcal{C}, \mathrm{T}}\right]^{\mathrm{T}}
$$

to be the state of the augmented dynamical system consisting of plant $\mathcal{P}$, controller $\mathcal{C}$ and the communication channel. By Eqs. (1), (7) and (13), we have

$$
x_{k+1}=A x_{k}-B L_{k} \hat{x}_{k}^{\mathcal{C}}+w_{k} .
$$

Above equation together with Eq. (13) determine the state evolution of $z_{k}$. The variable $x_{k}$ is now regarded as an output observation of the event-trigger, as it reveals just part of the augmented state $z_{k}$. The plant state $x_{k-\left(T_{1}+T_{2}\right)}$ and $\delta_{k-\left(T_{1}+T_{2}\right)}$ are known to the event-trigger, which implies that the controller state $\hat{x}_{k+1-T_{2}}^{\mathcal{C}}$ can be recalculated with knowledge of $q_{k-\left(T_{2}+T_{1}\right)}$ by Eq. (13) at time $k$. We define the information state

$$
\mathcal{I}_{k}^{\mathcal{E}}=\left\{\boldsymbol{X}^{k}, \boldsymbol{\delta}^{k-1}, \boldsymbol{Q}^{k-\left(T_{1}+T_{2}\right)}, \hat{\boldsymbol{X}}^{\mathcal{C}, k+1-T_{2}}\right\}
$$

which is regarded as the new argument for the eventtriggering law $\mathrm{f}_{k}$. In the following, we fix $T_{1}$ to be 1 and discuss the cases of $T_{2}=1$ and $T_{2}=2$. In the end, we state the main result for arbitrary $T_{1}, T_{2}$. For $T_{1}=1$, the augmented state variable $z_{k}$ reduces to $\left[x_{k}^{\mathrm{T}}, \hat{x}_{k}^{\mathcal{C}, \mathrm{T}}\right]^{\mathrm{T}}$. As $\hat{x}_{k}^{\mathcal{C}}$ may not be observed perfectly at the event-trigger, due to the delay in the acknowledgement, finding the optimal eventtrigger policy constitutes a problem with partial state information. It is well known that the conditional distribution $\mathrm{P}_{z_{k} \mid \mathcal{I}_{k}^{\mathcal{E}}}$ is a sufficient statistic for problems with partial state information, see e.g. Bertsekas (2005). The following paragraph is concerned with the calculation of $\mathrm{P}_{z_{k} \mid \mathcal{I}_{k}^{\mathcal{E}}}$. The variables, $x_{k}$ is within $\mathcal{I}_{k}^{\mathcal{E}}$ and therefore its conditional distribution is the Dirac distribution at $x_{k}$. For $\delta_{k-1}=0$, the variable, $\hat{x}_{k}^{\mathcal{C}}$ is Dirac distributed at $A \hat{x}_{k-1}^{\mathcal{C}}$. For $\delta_{k-1}=1$, the distribution of $\hat{x}_{k}^{\mathcal{C}}$ conditioned on $\mathcal{I}_{k}^{\mathcal{E}}$ is given by two values, $\left(A-B L_{k}\right) \hat{x}_{k-1}^{\mathcal{C}}$ and $A x_{k-1}-B L_{k} \hat{x}_{k-1}^{\mathcal{C}}$, occurring with conditional probability $\mathrm{P}_{q_{k-1} \mid \mathcal{I}_{k}^{\mathcal{E}} \text {. It turns }}$ out that $q_{k-1}$ is statistically independent of all random variables within $\mathcal{I}_{k}^{\mathcal{E}}$. Therefore, the conditional probability 
distribution $\mathrm{P}_{q_{k-1} \mid \mathcal{I}_{k}^{\mathcal{E}}}$ is given by the prior distribution of $q_{k-1}$, i.e. given by $\beta$.

It can be observed that the case of $T_{1}=T_{2}=1$ constitutes a special case, as the outstanding acknowledgement is statistically independent of the information available at the event-trigger at time $k$, i.e. $q_{k-1}$ is statistically independent of $\mathcal{I}_{k}^{\mathcal{E}}$. In contradistinction to this fact, observations at the scheduler can enhance the estimation of outstanding acknowledgements in case of $T_{2}>1$. This motivates us to consider $T_{2}>1$. In order to determine the conditional probability distribution of outstanding acknowledgements given $\mathcal{I}_{k}^{\mathcal{E}}$, we apply results established in Matei et al. (2008). There, Markovian jump linear systems were considered and least-squares state estimators were derived assuming delayed observations of outputs and the discrete mode of the system. For our underlying system, we regard outstanding acknowledgements as our discrete modes to be estimated. These are given by $\boldsymbol{Q}_{k-T_{2}}^{k-1}$. Applying results from Matei et al. (2008), it can be shown that the optimal estimator of the state $z_{k}$ is in general a nonlinear function of the observations at the eventtrigger. The estimator is nonlinear, as measurements are obtained before the discrete mode arrives at the eventtrigger. The advantage of the estimator is, that only recent observations have to be taken in account in order to derive the conditional probability distribution of $\boldsymbol{Q}_{k-T_{2}}^{k-1}$ given $\mathcal{I}_{k}^{\mathcal{E}}$. On the other hand, its computational complexity increases exponentially with the number of outstanding acknowledgements, which is in accordance with estimation problems for Markovian jump linear systems with unknown discrete states Bar-Shalom and Li (1993). In fact, the number of outstanding acknowledgements is limited by the round-trip time $T_{1}+T_{2}$ within the proposed system model. Hence, the calculation of the conditional probability distribution is computationally tractable for moderate round-trip times.

In the following, we present the conditional probability distribution of $\boldsymbol{Q}_{k-T_{2}}^{k-1}$ given $\mathcal{I}_{k}^{\mathcal{E}}$ for the case $T_{2}=2$.

Deriving the probability distribution of outstanding acknowledgements for arbitrary $T_{2}$ is performed similarly, but needs to consider more variables of past observations. Due to the fact that $q_{k-1}$ is statistically independent of observations $\mathcal{I}_{k}^{\mathcal{E}}$, the conditional distribution of $q_{k-1}$ is given by its prior distribution. Contrary to that, the estimation of the packet dropout occurring at time $k-2$ can be enhanced with the observation of $x_{k}$. Applying Matei et al. (2008) to the proposed framework, the conditional distribution is determined by

$$
\begin{gathered}
\mathrm{P}_{q_{k-2} \mid \mathcal{I}_{k}^{\mathcal{E}}}\left(\mathbf{q}||_{k}^{\mathcal{E}}\right)=\frac{\alpha\left(\mathbf{q},\left.\right|_{k} ^{\mathcal{E}}\right)}{\sum_{\ell \in 0,1} \alpha\left(\ell,\left.\right|_{k} ^{\mathcal{E}}\right)} \\
\alpha\left(\mathbf{q},\left.\right|_{k} ^{\mathcal{E}}\right)=\mathrm{P}\left[q_{k-2}=\mathrm{q}\right] f_{x_{k} \mid \mathcal{I}_{k-1}^{\mathcal{E}}, \mathbf{Q}^{k-2}\left(\mathrm{x}_{k}||_{k-1}^{\mathcal{E}}, \mathrm{Q}^{k-2}\right),}
\end{gathered}
$$

where $f_{x_{k} \mid \mathcal{I}_{k-1}^{\mathcal{E}}, Q^{k-2}}$ is the probability density function of $x_{k}$ given $\mathcal{I}_{k-1}^{\mathcal{E}}, \boldsymbol{Q}^{k-2}$. This function is a multivariate Gaussian function with covariance matrix $\Sigma_{w}$ and mean $m_{k}^{\mathrm{c}}$ depending on $q_{k-2}$ and $\delta_{k-2}$ :

$$
m_{k}^{\mathcal{E}}= \begin{cases}A x_{k-1}-B L_{k} A x_{k-2} & \delta_{k-2}=1 \wedge q_{k-2}=1 \\ A x_{k-1}-B L_{k} A \hat{x}_{k-2}^{\mathcal{C}} & \delta_{k-2}=1 \wedge q_{k-2}=0 \\ A x_{k-1}-B L_{k} A \hat{x}_{k-2}^{\mathcal{C}} & \delta_{k-2}=0\end{cases}
$$

We observe that

$$
\mathrm{P}_{q_{k-2} \mid \mathcal{I}_{k}^{\mathcal{E}}}=\mathrm{P}_{q_{k-2} \mid x_{k}, x_{k-1}, x_{k-2}, \hat{x}_{k-2}^{\mathcal{C}}, \delta_{k-2}}
$$

The calculation of $\mathrm{P}_{q_{k-2} \mid \mathcal{I}_{k}^{\mathcal{E}}}$ for a specific $q_{k-2}$ requires only the knowledge of $x_{k}, x_{k-1}, x_{k-2}, \hat{x}_{k-2}^{\mathcal{C}}$ and $\delta_{k-2}$.

Tracing back to our initial problem, that is the calculation of $\mathrm{P}_{z_{k} \mid \mathcal{I}_{k}^{\mathcal{E}}}$, we have already seen that the main issue is the determination of the conditional probability distribution of $\hat{x}_{k}^{\mathcal{C}}$ given $\mathcal{I}_{k}^{\mathcal{E}}$. By Eq. (13), $\hat{x}_{k}^{\mathcal{C}}$ can take four different values for $T_{2}=2$, depending on $q_{k-2}, q_{k-1}$ and $\mathcal{I}_{k}^{\mathcal{E}}$. Due to Eq. (14) and the fact that $q_{k-1}$ is statistically independent of $\mathcal{I}_{k}^{\mathcal{E}}$, the conditional distribution $\mathrm{P}_{z_{k} \mid \mathcal{I}_{k}^{\mathcal{E}}}$ is given by $x_{k}, \delta_{k-1}, x_{k-1}, x_{k-2}, \hat{x}_{k-2}^{\mathcal{C}}$, and $\delta_{k-2}$. In other words, the main result is that these variables are a sufficient statistic for solving (8), i.e. the optimal eventtriggering policy may be expressed as

$$
\mathrm{f}_{k}\left(\mathcal{I}_{k}^{\mathcal{E}}\right)=\tilde{\mathrm{f}}_{k}\left(\boldsymbol{X}_{k-2}^{k}, \boldsymbol{\delta}_{k-2}^{k-1}, \hat{x}_{k-2}^{\mathcal{C}}\right), \quad k=0, \ldots, N-1
$$

for and $T_{2}=2$. Variables with negative indices are omitted as arguments of $\tilde{\mathrm{f}}_{k}$. Similarly, it can be shown for arbitrary $T_{1}, T_{2}$ that

$$
\mathrm{f}_{k}\left(\mathcal{I}_{k}^{\mathcal{E}}\right)=\tilde{\mathrm{f}}_{k}\left(\boldsymbol{X}_{k-\max \left(T_{1}, T_{2}\right)}^{k}, \boldsymbol{\delta}_{k-\max \left(T_{1}+T_{2}\right)}^{k-1}, \hat{\boldsymbol{X}}_{k-T_{2}}^{\mathcal{C}}\right) .
$$

Considering the arguments of $\tilde{\mathrm{f}}_{k}$ as the new state, the underlying problem is a problem with full state information and we can apply the dynamic programming algorithm to find the optimal event-trigger law. In contrast to the initial problem within this subsection, the state does not increase in time, but only with $\max \left(T_{1}, T_{2}\right)$. Therefore, finding the optimal event-trigger $\tilde{f}$ is a numerically tractable problem for large horizon $N$ and moderate delays.

\section{NUMERICAL VALIDATION}

Suppose we have a scalar process $\mathcal{P}$ with $A=1, B=1$ and variances $\Sigma_{w}=1, \Sigma_{x_{0}}=1$. Parameters of the cost function are given by $Q=Q_{N}=1, R=10$ and $N=100$. Subsequently, we analyze the performance for three different packet dropout probabilities $\beta \in\{0,0,25,0.5\}$, forward delay $T_{1}=1$ and reverse delay $T_{2} \in\{1,2\}$. The proposed suboptimal algorithms are compared with the optimal time-triggered controller for various communication penalty $\lambda$. The time-triggered strategy does not need an acknowledgement channel. Therefore, it is independent of $T_{2}$. The optimal transmission timings of the timetriggered controller are calculated by the deterministic dynamic programming algorithm.

In addition, a lower bound on the cost is determined by assuming that the acknowledgement channel has no delay, i.e. $T_{2}=0$. For such situation, it has been shown in Molin and Hirche (2009) that the optimal event-based controller can be calculated efficiently. It is clear that this bound is not tight for $\beta>0$. It can not be achieved by any event-based controller, as it would impose to guess always correctly, whether a packet dropout occurred before the actual acknowledgement is received. A comparative study is illustrated in Fig. 2 for different packet dropout probabilities.

In all three cases, we observe that the dropout estimation strategy outperforms the optimal time-triggered algorithm and approaches the lower bound very closely. In fact, the 

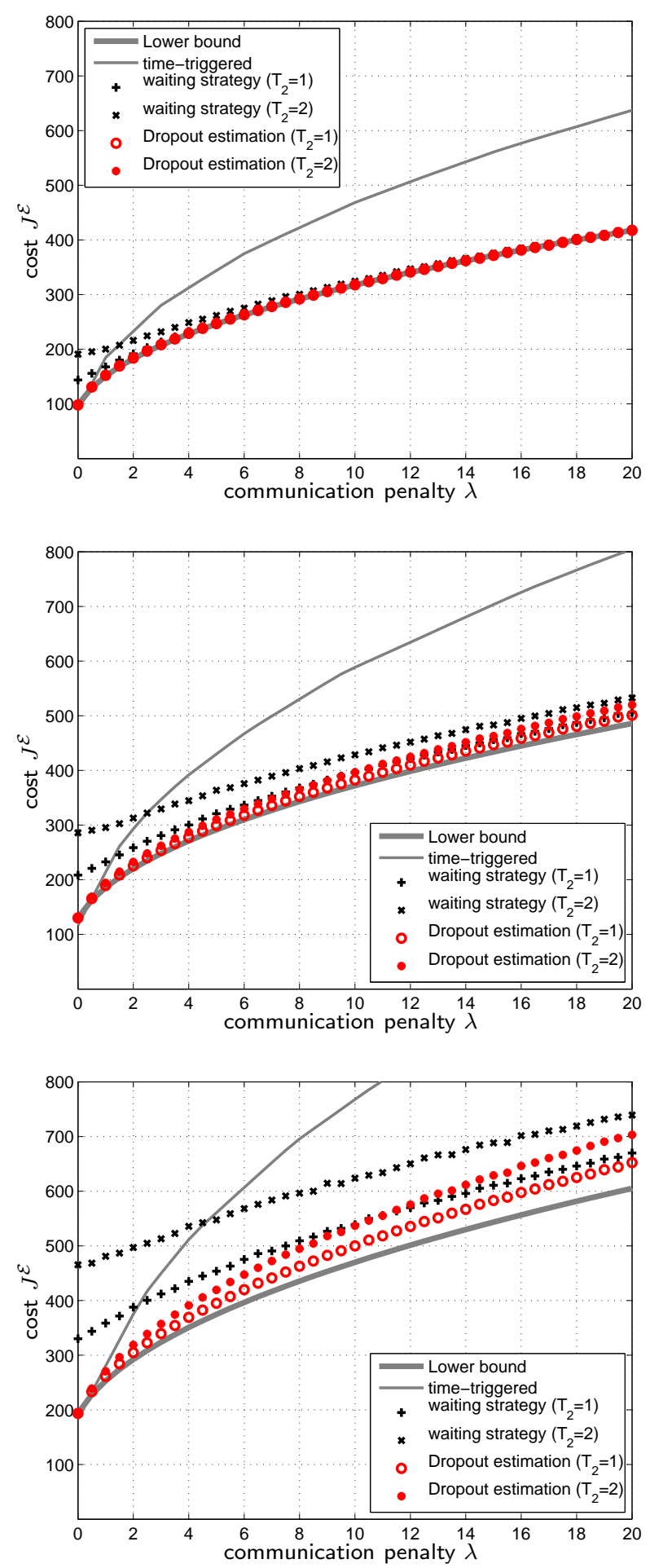

Fig. 2. Performance comparison: without packet dropouts (top); for packet dropout probability $\beta=0.25$ (center); for packet dropout probability $\beta=0.5$ (bottom).

performance of the dropout estimation strategy and the lower bound are equal for $\beta=0$, because there is no need in estimating packet dropouts for arbitrary $T_{2}$. The waiting strategy outperforms the optimal time-triggered algorithm for $\lambda>5$. Delay $T_{2}$ plays a crucial role for the performance of the waiting strategy. In case of low communication penalty $\lambda$, the time-triggered algorithm is better suited than the waiting strategy. This is a natural consequence as transmissions prevail during horizon $N$ for small $\lambda$, but the waiting strategy does not permit to transmit more than $\left\lceil\frac{N}{T_{1}+T_{2}}\right\rceil$ times. Evidently, the dropout estimation strategy shows better performance than the waiting strategy at the cost of additional computations.

\section{CONCLUSION}

By considering a lossy communication network with delays and an acknowledgement mechanism, this paper proposes two types of optimal event-based control design under two different assumptions. Despite of their computational benefits compared to the optimal solution, it turns out that both algorithms approach the optimal solution very closely. The numerical validation indicates significantly that the suboptimal algorithms outperform time-triggered control design in the presence of communication penalties. Future work comprises the consideration of output systems and the extension of the communication model to erasure channels with time-varying time-delay and Markovian packet dropouts.

\section{REFERENCES}

Åström, K.J. (2006). Introduction to Stochastic Control Theory. Dover Publications.

Åström, K. and Bernhardsson, B. (2002). Comparison of Riemann and Lebesgue sampling for first order stochastic systems. Decision and Control, 2002, Proceedings of the 41st IEEE Conference on, 2, 2011-2016.

Bar-Shalom, Y. and Li, X. (1993). Estimation and tracking- Principles, techniques, and software. Norwood, MA: Artech House, Inc.

Bertsekas, D.P. (2005). Dynamic programming and optimal control. Vol. I. 3rd ed., Athena Scientific, Belmont, MA.

Bommannavar, P. and Başar, T. (2008). Optimal control with limited control actions and lossy transmissions. Decision and Control, 2008 47th IEEE Conference on.

Cervin, A. and Henningsson, T. (2008). Scheduling of event-triggered controllers on a shared network. In Proc. 47th IEEE Conference on Decision and Control.

Matei, I., Martins, N., and Baras, J. (2008). Optimal state estimation for discrete-time Markovian Jump Linear Systems, in the presence of delayed mode observations. In American Control Conference, 2008, 3560-3565.

Molin, A. and Hirche, S. (2009). On LQG joint optimal scheduling and control under communication constraints. In Proc. 48th IEEE Conference on Decision and Control.

Rabi, M. and Johansson, K.H. (2009). Scheduling Packets for Event-Triggered Control. In Proc. European Control Conf. (ECC).

Schenato, L., Sinopoli, B., Franceschetti, M., Poolla, K., and Sastry, S. (2007). Foundations of control and estimation over lossy networks. Proceedings of the IEEE, 95(1), $163-187$.

Witsenhausen, H.S. (1968). A counterexample in stochastic optimum control. SIAM Journal on Control, 6(1), 131-147.

Xu, Y. and Hespanha, J. (2004). Optimal communication logics in networked control systems. In Proc. 43th IEEE Conference on Decision and Control, 3527-3532. 